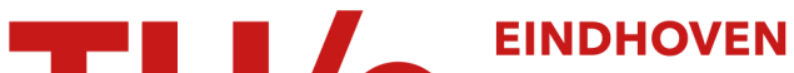 UNIVERSITY OF TECHNOLOGY
}

\section{Fokker-Planck equation for chemical reactions in plasmas}

\section{Citation for published version (APA):}

Longo, S., van de Sanden, M. C. M., \& Diomede, P. (2019). Fokker-Planck equation for chemical reactions in plasmas. Rendiconti Lincei. Scienze Fisische e Naturali, 30(1), 25-30. https://doi.org/10.1007/s12210-01900784-x

DOI:

10.1007/s12210-019-00784-x

Document status and date:

Published: $13 / 03 / 2019$

\section{Document Version:}

Accepted manuscript including changes made at the peer-review stage

\section{Please check the document version of this publication:}

- A submitted manuscript is the version of the article upon submission and before peer-review. There can be important differences between the submitted version and the official published version of record. People interested in the research are advised to contact the author for the final version of the publication, or visit the $\mathrm{DOI}$ to the publisher's website.

- The final author version and the galley proof are versions of the publication after peer review.

- The final published version features the final layout of the paper including the volume, issue and page numbers.

Link to publication

\section{General rights}

Copyright and moral rights for the publications made accessible in the public portal are retained by the authors and/or other copyright owners and it is a condition of accessing publications that users recognise and abide by the legal requirements associated with these rights.

- Users may download and print one copy of any publication from the public portal for the purpose of private study or research.

- You may not further distribute the material or use it for any profit-making activity or commercial gain

- You may freely distribute the URL identifying the publication in the public portal.

If the publication is distributed under the terms of Article 25fa of the Dutch Copyright Act, indicated by the "Taverne" license above, please follow below link for the End User Agreement:

www.tue.nl/taverne

Take down policy

If you believe that this document breaches copyright please contact us at:

openaccess@tue.nl

providing details and we will investigate your claim. 


\title{
Fokker-Planck equation for chemical reactions in plasmas
}

\author{
Savino Longo ${ }^{1}$, Mauritius C. M. van de Sanden ${ }^{2}$, Paola Diomede ${ }^{2}$
}

${ }^{1}$ Dipartimento di Chimica, Università degli Studi di Bari, via Orabona 4, 70126 Bari, Italy and CNRNanotec, Bari, Italy.

${ }^{2}$ Center for Computational Energy Research, DIFFER - Dutch Institute for Fundamental Energy Research, De Zaale 20, 5612 AJ Eindhoven, the Netherlands

Keywords: reactions in plasma, Fokker-Planck equation, numerical methods

\begin{abstract}
In the past few years, a new approach has been experimented for the calculation of the populations of vibrational levels of molecules involved in chemical reactions in ionized gases. The approach, that can be defined as neo-diffusive, is based on the use of numerical and functional techniques to solve a Fokker-Planck equation in the space of internal energy. The transport coefficients are calculated from the rate coefficients of the reactions between molecules in different vibrational states. It represents a conceptually different alternative to the much-used state-to-state (STS) approach, based on the solution of the Master Equation. The neo-diffusive approach differs from a similar Fokker-Planck equation-based approach used in the 70s and the $80 \mathrm{~s}$ of the past Century since exact numerical solutions are used. In this work, the state of the art and perspectives of this new approach are analyzed. Is it pointed out that, while the neo-diffusive method allows us to calculate populations of vibrational levels and reaction rates with much less computational effort than methods based on the Master equation, much important is that this method provides a powerful basis for intuition, allowing to highlight effects otherwise obscured by the complex network nature of the STS approach.
\end{abstract}

\section{Introduction: Vibrational kinetics and the state-to-state approach}

In partially ionized gases, thanks to the presence of free electrons which can reach energies of the order of electron volts or even much higher in the presence of strong electric fields, chemical reactions occur, which are induced by those energetic electrons and which are important both from a fundamental and from an application point of view. Among the chemical reactions that are important from a fundamental point of view, there are those in the interstellar medium or in highly ionized planetary 
atmospheres. From an applicative point of view, one can cite all the reactions for the production of species active in the context of materials processing and the development in the past of molecular gas lasers such as carbon dioxide and excimer lasers. A particularity of many of these reactions is the fact that the reaction rate is conditioned by the specific properties of the various excited states of atoms or molecules involved. A well-known example is the reaction for the production of negative ions starting from vibrationally excited hydrogen molecules, also called dissociative attachment: The rate coefficient of this reaction increases tremendously with the vibrational level peaking at about the fifth-seventh level [1] with values four orders of magnitudes higher than the one for the ground vibrational state. Another peculiarity of partially ionized gases subjected to electric fields is that energy levels of atoms and molecules are populated by specific mechanisms related to energy reservoirs not in equilibrium between them: for this reason, the excited state populations do not follow the distribution valid in the equilibrium case, that is, the Boltzmann distribution $[2,3]$. This leads to the state-to-state (STS) description. This last is based on the idea that a molecule in any specific quantum state reacts with a specific reaction rate with other species to produce different excited states and other reaction products [2-5]. As a consequence, the only way to describe in a quantitatively correct way chemical reactions occurring in ionized gases is to calculate these populations as a function of time, by solving appropriate kinetic equations: the most widely used is the Master equation, a system of ordinary differential equations linking the distribution of populations of different levels. The description of numerous kinds of plasma by this method produced excellent results in terms of comparison with experimental results, with the perspective to optimize the process for systems of applicative interest and it has also greatly contributed to the understanding of ionized gas kinetics by highlighting the complex and detailed pathways that produce variation in time of the chemical composition of the system, and cannot be replaced in any effective way by simpler approaches disregarding these distributions $[2,3]$. On the other hand, the Master equation is a very complex equation in that it has as many unknowns as the states considered in the model and a right-hand side that includes a large number of terms, each of which is connected to a single STS chemical process. It is evident that the Master equation involves some serious difficulties when it is applied to the practical resolution of the kinetic problem, a factor that clearly limits the application of this methodology in cases of systems characterized by very complex vibrational ladders: the number of potential level-to-level transitions for a system of $n$ levels increases with the square of $n$ and therefore very quickly. A second factor is a limit to the intuition that can be gained from 
results of kinetic simulations. In this approach, a net of flows is created between possible discrete levels from which it is difficult to extract insights. In fact, the a posteriori analysis of results based on the numerical solution of the Master equation normally covers a considerable part of the corresponding scientific works and it is often analyzed separately in later works.

\section{The continuous approach}

In order to solve these problems, in the past few years our group has considered a line of analysis that had been much used in the past, in particular in the 70s and 80 s of the past Century, by physical chemists interested in this type of problems and similar problems that imply numerous internal levels such as atomic recombination in ionized gases [6-10]. This approach, that can be called a continuous approach or, as we propose, a neo-diffusive approach, is based on the idea of replacing the discrete internal levels with a continuum: this is of course an approximation but in practical terms usually a good one, even when the number of levels is not very high (Figure 1). For this purpose, a function, $F(\varepsilon, t)$, is defined as the extension to a continuum of energy of the function describing the populations of the corresponding discrete levels as a function of the internal energy. The internal energy $\varepsilon$ is evaluated

starting from the zero-point energy of the corresponding system of oscillators and then defined up to the energy needed to reach the dissociation threshold. This function $F$ is the solution of the FokkerPlanck equation:

$$
\frac{\partial F}{\partial t}=\frac{\partial}{\partial \varepsilon}\left(-a F+b \frac{\partial F}{\partial \varepsilon}\right)
$$

which is a partial differential equation of the second order. In the Fokker-Planck equation, two so-called transport coefficients appear: the drift coefficient, indicated here by $a$ and the diffusion coefficient, indicated here by $b$. These two coefficients are not constants but functions of the internal energy. In some cases, it may be necessary to add to the Fokker-Planck equation either sink or source terms. For example, this happens when a chemical process destroys molecules with intermediate internal energy. However, if the destruction process happens when the internal energy exceeds its maximum values, 
like in molecular dissociation, the process is described by imposing an appropriate boundary condition to eq.(1) and no sink/source term is requested.

The approach is interesting and also practical to use because there are numerous techniques for numerical and, in a few cases, analytical solutions of the Fokker-Planck equation which is, indeed, one of the most important equations of mathematical physics. The other factor that makes the approach of practical use, is the availability of methods that allow to explicitly calculate drift and diffusion coefficients starting from expressions of probabilities that the system undergoes transitions between two states: these expressions are well known and are found in textbooks dedicated to the theory of stochastic processes, such as the book by Van Kampen [11]. Specific expressions for the vibrational kinetics of molecules are found in a book by Fridman [10]. For example, a process of the form $A+X(\varepsilon)$ $\rightarrow \mathrm{A}+\mathrm{X}(\varepsilon+\delta \varepsilon)$ where $\mathrm{A}$ is a reaction partner, gives a contribution to the drift coefficient given by $\Delta \mathrm{a}=$ $\mathrm{k}_{\mathrm{A}} \delta \varepsilon$, where $\mathrm{k}$ is the rate coefficient of the process and $\mathrm{n}_{\mathrm{A}}$ is the number density of $A$. Since the coefficients $k$ are defined only for quantized energy levels, they must be interpolated onto the continuum energy axis. As can be seen from this example, there is no limitation of the method to monoquantum processes, although $\delta \varepsilon$ must be much smaller than the dissociation energy, otherwise the Fokker-Planck description is not appropriate.

These expressions allow an intuitive interpretation: for example, if a chemical process involving the molecule under examination and a second species mainly produces a decrease in internal energy, i.e. the passage from an energy level to a lower one, this process will contribute (negatively) to the drift coefficient, while giving only a small contribution to the diffusion coefficient: an example in the vibrational kinetics is the so-called VT process in which the impact of a molecule in a vibrationally excited state with a molecule or atom of the medium dissipates the energy corresponding to one or more quanta in the form of kinetic energy and therefore of heat. On the other hand, there are processes involving excited molecules and atoms that can lead both to an increase and to a decrease of internal energy: if these two outcomes are equally likely, it is easy to understand that the process produces a diffusion phenomenon and therefore contributes to the diffusion coefficient. In this case there may be a also contribution to the drift coefficient due to the anharmonicity of the molecular oscillators: the difference in energy between a level and a higher level is lower, although slightly, than the difference in energy between the same level and a lower level. Contribution to $a$ and $b$ of the same process are related by detailed balance. In the case of vibrational energy distributions, the anharmonicity must be 
accounted for. For example, in an ensemble of molecules in a thermal gas at temperature $\mathrm{T}_{\mathrm{g}}$ and when only vibrational energy exchanges (or VV) process are important, the Treanor distribution must be obtained at the steady state [7-10]. To grant this, the adimensional quantity Tvavv/bvv must satisfy the relation

$$
\frac{T_{V} a_{V V}}{b_{V V}}=\frac{2 x_{e} \varepsilon}{\hbar \omega} \frac{T_{V}}{T_{g}}-1
$$

Where $T_{v}$ is the vibrational temperature, $x_{e}$ the constant of anharmonicity and $\hbar \omega$ is the energy difference between the ground and first excited levels. $T_{v}$ is defined as the temperature that provides the best approximation of lowest levels populations using a Treanor distribution.

As it has been demonstrated in [12], eq.(2) together with a dissociative boundary condition explains the most relevant features of the energy distribution, including the formation of super-thermal tails as in the case of $\mathrm{CO}_{2}$ discussed later. In reality the details of the chemical processes of the molecular interaction, together with the principle of detailed balance, lead to a variegated set of probabilities to gain or lose energy for which drift and diffusion coefficients will depend in a more or less complex way on the internal energy and also on the values of global system parameters such as the gas temperature and the vibrational temperature of the oscillators.

\section{Application to $\mathrm{CO}_{2}$}

In two recent works $[12,13]$, we have already applied this methodology to the study of plasmas produced using carbon dioxide as feed gas: the reason why this study has recently become of great interest is not just applicative, but even of possible impact for the human society as a whole: the transformation of carbon dioxide into more chemically reactive species such as carbon monoxide and oxygen [14]. This process can be a way to solve the problem of the accumulation of anthropogenic greenhouse gases in the Earth's atmosphere and recently it has also been proposed that this process of transformation, or as it is sometimes called, of plasma activation of carbon dioxide, may be a key to the colonization of Mars [15], by solving the problem posed by the Mars atmosphere, constituted almost exclusively of carbon dioxide. The process via plasma becomes very attractive thanks to the proposal 
to operate the plasma with renewable energy, in particular solar or wind energy. It is also interesting to note that the study of the dissociation of carbon dioxide in ionized gases was one of the most important application of the diffusion-based approach in the 70s.

Our approach differs from the original diffusive approach, since it employs numerical calculation methods which have in the meantime become inexpensive and widely available. For example, we used the Diffusion Monte Carlo method which is not particularly slow if the many techniques available for variance reduction are used. Details of this approach have been reported in [12]. In the case of stationary problems, we have proposed to use semi-analytical techniques that are obviously extremely fast, in particular, in the work [13], a shooting technique similar to those used for the solution of the Schrödinger equation in one dimension has been employed: starting from the zero-point energy, this technique traces the function $F$ based on a guess of the value of the functional $J$, the total reactive flux, that in turn depends on the unknown solution. $J$ is actually the number of dissociation events per molecule and unit time. The estimate of $J$ is then modified at the end of each solution, progressively reducing the difference between $J$ and its microscopic estimate based on rates of dissociation reactions of levels close to the continuum. The neo-diffusive approach can therefore be considered a functional method. It has been shown that this approach reproduces results obtained by the STS technique. Nevertheless, it differs profoundly from STS in its conceptual approach and methodology, thus providing a very useful alternative point of view as it will be discussed later. In Figure 2(left), the transport coefficients calculated by using the method described in [13] are shown. Using these values, the vibrational distribution function (VDF) in Figure 2(right) is obtained. Three typical regions are observed: the bulk, a quasi-Boltzmann distribution at low energy before the onset of vibrational quanta exchange processes, the plateau due to these last, and the final fall at high energy due to the effect of VT processes. When these results are calculated for parameter values for which STS results are available, a good agreement is usually observed $[12,13]$.

\section{Comparison with the discrete state-to-state approach}

It is evident that the proposed method, compared with the traditional method based on the solution of the Master equation, presents some drawbacks: to begin with, it is based on an approximation, whereas the Master equation is in principle an exact setting; besides, the solution of problems in 
differential form and functional problems requires a mathematical knowledge greater than that required for the Master equation-based approach. It is also to be observed that, contrary to the limit approaches used in plasma physics, like the passage to a high plasma parameter under appropriate conditions, the vibrational quantum cannot be reduced, therefore the diffusive approach is always an approximate one. These disadvantages, however, are balanced by important advantages: the solution of a differential problem using the appropriate methods can be extremely efficient and, in some cases, it is even possible to propose explicit analytical solutions even for fairly wide classes of problems. Indeed, it is well known that this approach has been used in the past primarily for the purpose of avoiding the use of computing machines that were still poorly distributed or expensive in particular in the former Soviet Union where the diffusion-drift formalism has been widely developed. Another advantage is the compression or reduction of data needed to solve the problem: in fact, where the STS approach requires a complete set of kinetic coefficients that coherently copy the set of possible STS transitions, the continuous method simply requires the specification of two functions, the drift and diffusion coefficient. Although it is true that the explicit calculation of these two functions still requires knowledge of the rate coefficients of the involved reactions, the fact that the final result is in the form of two functions that must be continuous and differentiable, allows us to reduce greatly the uncertainties related to the complete coverage of the transitions set. In practice, in many cases the needs related to the mathematical properties of these functions and the knowledge of the main rate coefficients allows us to fix almost completely the functions under examination and therefore to greatly reduce the well-known possibilities of errors that can creep into a STS calculation due to the lack of data that often produces discontinuities and inconsistencies in STS rate coefficient sets. Moreover, from the point of view of the transportability of the data, that is, the creation and maintenance of the data set necessary to make the calculation program work, it is clear that the use of only two functions, that can be further reduced using interpolation coefficients, allows great practical savings, also in the management of calculation programs. Indeed, the typical STS database including hundreds or thousands of data is reduced to a set of a few, typically not more than twenty, interpolating expressions that can be safely hardcoded in the program source.

Of course, replacing a Master Equation by a Fokker-Planck one is not always a panacea. For example, when studying transients $(\partial F / \partial t \neq 0)$, the numerical solution of the Fokker-Planck may be difficult if the drift term is dominant over the diffusion term. This problem has been addressed in [12] and a solution 
attempted, in the form of a Monte Carlo approach with efficient variance reduction. If the steady-state distribution is sought, fast methods can be used to obtain the exact solution [13]. However, as discussed in the next section, the point is not to select the "best method" from Master Equation and FokkerPlanck, but to gather insight on the overall reaction kinetics by adopting the point of views of both approaches.

\section{The heuristic value of the continuous approach}

So far, we have discussed practical advantages of the neo-diffusion method, but it is important to note that the greatest benefits are obtained in terms of interpretation and intuition. In fact, it is very beneficial to discuss the complex evolution of the molecular levels using reaction rates from level to level, which can be described as a complex graph, using instead the tools of transport theory in the neodiffusive approach. Let us give an example of results of this kind that were recently obtained: in the case of the carbon dioxide dissociation, it has often been stated that the reason why the best results are obtained in plasma reactors in which the gas is cooled considerably, for example by adiabatic expansion, is that lowering the temperature reduces the specific rate of molecular processes (like the VT) which dissipate vibrational energy from the levels closer to the continuous. Our approach shows, on the contrary, that the vibrational kinetics is controlled by the temperature ratio $T_{v} / T_{g}$ as shown by eq.2 above. This idea has been used successfully in recent works in which the possibility of using plasmas in an atmosphere of carbon dioxide like that of Mars is used and to evaluate a colonization scenario [15]. Another important insight is that, unless the vibrational temperature is not very low, a change of sign of the drift coefficient is observed, from negative to positive, at a defined vibrational energy (see again eq. 2 and Figure 2 here). Because of this change of sign, a flow towards dissociation is established. Therefore, molecules that have exceeded this value of vibrational energy can only dissociate: We then understand that even if there are reactions that have the ability to dissipate the vibrational energy from levels close to continuum, those reactions, pushing the molecules back in a certain sense along the energy scale, can only produce an accumulation of molecules at slightly lower energies but cannot change the overall reaction rate. The analogy with the communicating vessels illustrated in Figure 3, inspired by the artwork of J. Sonnabend in [17], is useful: the flow of liquid through the vessels is constant; if one of the ducts is narrowed, this increases the volume of the liquid 
in the tank immediately upstream of the restricted duct, in turn increasing the flow velocity of the liquid and keeping the flow rate constant. This is a special case of the so-called buffer effect that already many years ago was studied by one of the authors in the case of kinetic models of ionized gases [16]. We can see how the application of concepts of transport theory and fluid dynamics to chemical reactions involving excited levels of molecules allows us to find interpretative keys that can direct research towards methods of greatest impact and simplify the description of phenomena as a whole.

\section{Conclusions}

In this work, it has been shown how the application of the Fokker-Planck equation to chemical reactions in plasma phase can strongly facilitate the study of these reactions in various ways. Our approach, that, in analogy to other methodologies applied in the study of ionized gases, we propose to name neodiffusive, is based a huge heritage of concepts and mathematical techniques developed decades ago, but interprets them differently by widely using numerical and functional methodologies to extract concrete results for real cases from the formalism. Approximations usually adopted in previous studies are no longer necessary. From a computational point of view, this method is currently being developed in order to produce results as close as possible to those obtained by solving the Master equation but, especially in the stationary case, using much less calculation time: this is possible thanks to the possibility of solving the equations in a semi-analytical way and can find application in multi-physical models of real systems and reactors. In this context, it is also worth mentioning here the prospect of using this method for kinetic and vibrational descriptions with several degrees of freedom, since the STS approach in such a case is hopeless, while there are many powerful techniques already experimented to solve multidimensional diffusion problems, like the already cited diffusion Monte Carlo. However, the main advantage of the application of this method is in the field of intuition: in fact, the possibility of using concepts of transport theory in describing a multi-level kinetics allows us to highlight the effects that lead the system towards a given outcome. In this way it is possible to grasp the essence of the phenomena and to concentrate on aspects that mostly influence the rate of the overall chemical reaction that is investigated.

\section{Acknowledgements}


This work is part of the Shell-NWO/FOM initiative 'Computational sciences for energy research' of Shell and Chemical Sciences, Earth and Life Sciences, Physical Sciences, FOM and STW, project number 14CSTT02, "Fast and accurate computational approaches to molecular dissociation in non-equilibrium plasmas: the case for $\mathrm{CO}_{2}$ dissociation". 


\section{References}

\section{References}

[1] Allan, M. and Wong, S. F. Effect of vibrational and rotational excitation on dissociative attachment in hydrogen. Phys. Rev. Lett. 1978, 41, 1791.

[2] Diomede, P; Bruneau, B.; Longo, S.; Johnson, E.; Booth, J.-P. Capacitively coupled hydrogen plasmas sustained by tailored voltage waveforms: vibrational kinetics and negative ions control. Plasma Sources Sci. Technol. 2017, 26, 075007.

[3] Capitelli, M. et al. Non-equilibrium plasma kinetics: a state-to-state approach. Plasma Sources Sci. Technol. 2007, 16, S30.

[4] Levine, R. D., Molecular reaction dynamics; Cambridge University Press: New York, 2009.

[5] Steinfeld, J. I.; Francisco, J. S.; Hase, W. L., Chemical kinetics and dynamics, Vol. 3., Chap. 9; Englewood Cliffs (New Jersey): Prentice Hall, 1989.

[6] Lifshitz, E. M. and Pitaevskii, L. P., Landau and Lifshitz, Physical Kinetics: Course of Theoretical Physics, 1st ed., Vol. 10, Chap. 2; Pergamon Press, 1981.

[7] Brau, C. A. Classical Theory of Vibrational Relaxation of Anharmonic Oscillators. Physica 1972, 58, 533-553.

[8] Zhdanok, S. A.; Napartovich, A. P.; Starostin, A. I. Establishment of the vibrational distribution of diatomic molecules. Sov. J. Exp. Theor. Phys. 1979, 49, 66.

[9] Rusanov, V. D.; Fridman, A. A.; Sholin, G.V. The Physics of a Chemically Active Plasma with Nonequilibrium Vibrational Excitation of Molecules. Sov. Phys. Usp. 1981, 24, 447-474.

[10] Fridman, A. Plasma Chemistry; Cambridge University Press: New York, 2008.

[11] Van Kampen, N. G. Stochastic Processes in Physics and Chemistry; Vol. 1, Elsevier: Amsterdam, The Netherlands, 1992.

[12] Diomede, P.; van de Sanden, M. C. M. and Longo, S. Insight into $\mathrm{CO}_{2}$ Dissociation in Plasmas from Numerical Solution of a Vibrational Diffusion Equation. J. Phys. Chem. C 2017, 121, 19568-76.

[13] Diomede, P.; van de Sanden, M. C. M. and Longo, S. Vibrational Kinetics in Plasma as a Functional Problem: A Flux-Matching Approach. J. Phys. Chem. A 2018, 122, 7918-7923.

[14] Goede, A. and van de Sanden, M. C. M., $\mathrm{CO}_{2}$-Neutral Fuels, Europhys. News 2016, 47, 22-26. 
[15] Guerra, V.; Silva, T.; Ogloblina, P.; Grofulović, M.; Terraz, L.; Lino da Silva, M.; Pintassilgo, C. D.; Alves, L. L. and Guaitella, O. The Case for in Situ Resource Utilisation for Oxygen Production on Mars by Non-Equilibrium Plasmas. Plasma Sources Sci. Technol. 2017, 26, 11 LT01.

[16] Longo, S.; Gorse, C. and Capitelli, M. Open problems in the XeCl laser physics. IEEE Trans. Plasma Sci. 1991, 19, 379-386.

[17] Waddington, C. H. Tools for thought. Cape., 1977. 


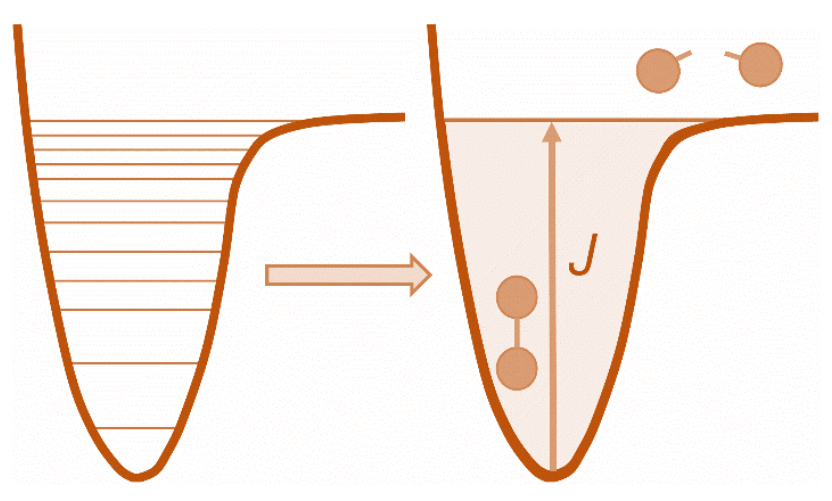

Fig. 1 Illustration of the neo-diffusion approach: on the left, an artistic idea of the detailed internal energy levels of a molecule are shown superimposed to a typical, Morse-like interatomic potential. On the right, the continuum of internal energy which replaces these levels, with the important role played by the constant value of the total flow $J$ outlined (adapted from [13])
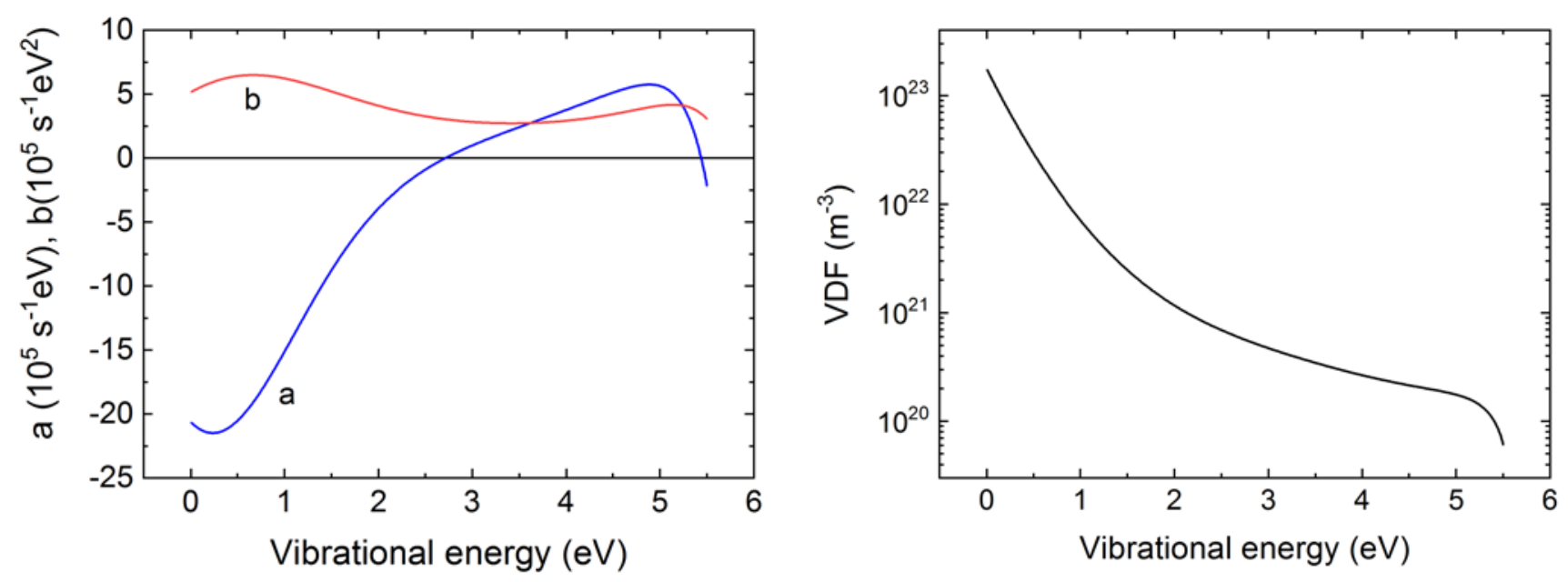

Fig. 2 Transport coefficient $a$ and $b$ (left) and vibrational distribution function (right) calculated for the asymmetric stretching of $\mathrm{CO}_{2}$ for $T_{v} / T_{g}=9.7$. Note the sign inversion of the drift discussed in the text, occurring here at $2.7 \mathrm{eV}$. 


\section{Inflow extra process}

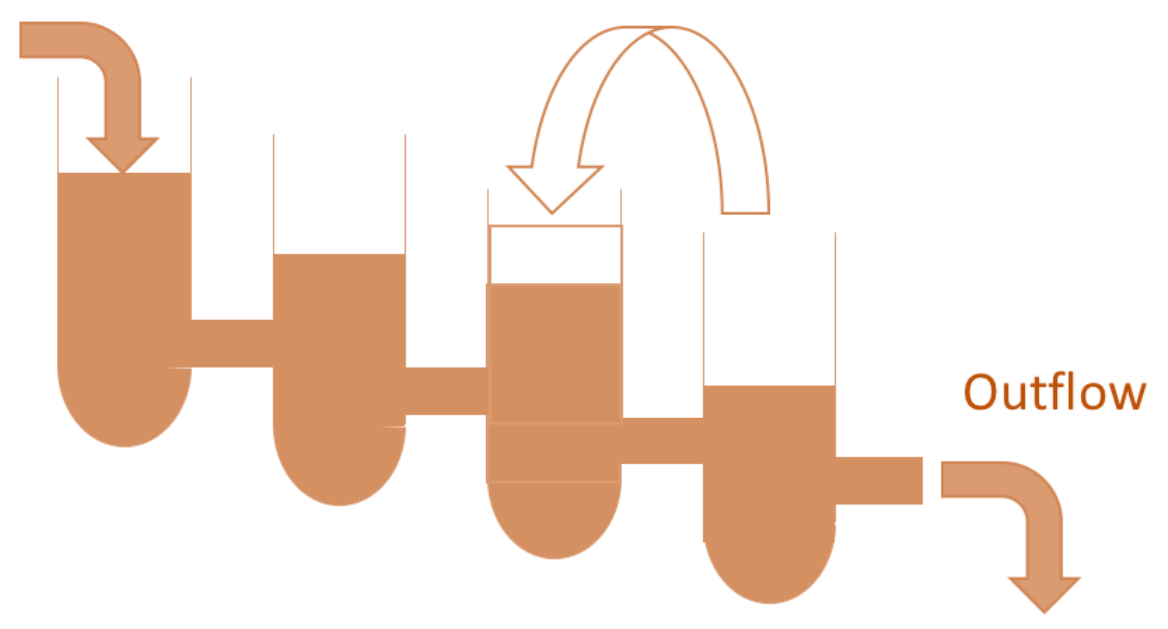

Fig. 3 Analog illustration of the buffer effect in the population kinetics. Water is flowing downhill from the uppermost vessel. The water flow is constant: this determines the level of the water in each vessel. If a duct diameter is changed, the water level uphill will change, but the outflow will always equal the inflow at the steady state. The same happens if water is continuously extracted from one vessel and poured into another uphill. This system of communicating vessels is an illustration of the general behavior of large classes of systems and in particular applies to the vibrational kinetics under positive drift regimes: in this case, different containers correspond to vibrational levels, water levels to populations, duct diameters to rate coefficients, and water transfer to a vibrational quenching process. 